\title{
Bubble Coalescence and Breakup in Turbulent Bubbly Wake of a
}

\section{Ventilated Hydrofoil}

Ashish Karn ${ }^{1,2}$, Siyao Shao ${ }^{1,2}$, Roger E A Arndt ${ }^{1}$, Jiarong Hong ${ }^{1,2, *}$

1. Saint Anthony Falls Laboratory, $23^{\text {rd }}$ AVE SE, University of Minnesota, Minneapolis, MN, USA 55414.

2. Department of Mechanical Engineering, University of Minnesota, Minneapolis, MN, USA 55455.

* Email addresses of the corresponding author: jhong@umn.edu

\begin{abstract}
Auto-venting turbines have been proposed as a promising solution to the problem of low oxygen content in the discharged downstream water of an electric power plant. The current design of these turbines relies primarily on computational simulation. The experimental studies that focus on the physical processes occurring in turbulent bubbly wake are urgently needed to improve the performance of these simulations in predicting the bubble size distribution behind auto-venting turbines. Therefore, in the current study, we conducted detailed experimental investigations into the bubble size distributions in the wake of a ventilated hydrofoil. The mean bubble statistics is measured at different liquid velocities and air entrainment rates, and then the variation in mean bubble statistics is studied at different downstream locations in the wake. The bubble size distributions at different downstream locations have revealed the presence of distinct coalescence-dominant and breakup-dominant regimes. Analytical expressions are derived for the prediction of maximum stable diameter and Sauter mean diameter of bubbles, in the breakup and coalescence regimes, respectively. The observations from high speed imaging provide support for the measurements of bubble statistics, and physical insights into different mechanisms of bubble breakup and coalescence in turbulent wake. It is hoped that these insights will aid in developing generic model of bubble size distribution, and will help researchers improve bubbly flow simulations for auto-venting turbines.
\end{abstract}

Keywords: Bubble coalescence; Bubble breakup; Bubbly wake; Auto-venting turbines, Hydroturbine aeration; Shadow image velocimetry. 


\section{Introduction}

Hydroelectric power, although one of the major contributors to the renewable non-polluting energy source, has a potential disadvantage [1]. In the hydropower facilities, usually the penstocks feeding the turbines are installed deep in the reservoirs. At such large depth within the impoundments of water, the oxygen concentration is significantly low. This water, which is low in dissolved oxygen, when released, adversely affects the downstream ecosystem, including the aquatic flora and fauna [2]. As a result, increasingly stringent water quality standards have been posed on the hydropower plants to improve the dissolved oxygen (DO) levels in the downstream water. Such strict requirements have impelled the development of aeration techniques to replenish the oxygen concentration in the water.

Auto-venting turbines (AVT) have been proposed as a promising solution to the problem of low oxygen content in the discharged downstream water of an electric power plant $[3,4]$. In an AVT, air is drawn into the water at naturally occurring low pressure points in turbines leading to increased dissolved oxygen levels in the tailraces below hydroelectric dams. However, although the aerated hydroturbines improve the water quality, yet the increasing air ventilation in the turbine has a detrimental effect on the turbine efficiency due to subsequent hydraulic losses. Accordingly, the design and operation of such AVTs have to fulfill the twin objectives of increased DO levels, and minimal drop in turbine efficiency. Currently, much of the design of auto-venting hydroturbines is performed with computational fluid dynamics (CFD) simulations. The information available to test and verify the performance of these simulations as regards bubble size distribution is scarce. Also, these simulations are challenging since capturing the evolution of bubble size distribution in the wake accurately requires an understanding of the process of air entrainment and other physical processes occurring in the turbulent bubbly wake. 
The air entrainment in such a hydroturbine can be carried out by opening a port connected to the low-pressure regions in the turbine. Evidently, when air is entrained into the wake, it breaks into individual bubbles because of the presence of turbulent velocity fluctuations in the wake. Further, the collision of bubbles might also produce bubbles with increased size due to coalescence. In a turbulent and dispersed liquid flow, breakup and coalescence processes occur continuously and interplay between these two processes determines the bubble size distribution. Finally, bubbles attain a stable size due to the establishment of a local dynamic equilibrium between these two processes. However, the underlying mechanism in which the entrained air breaks into bubbles and the change of bubble size in the turbulent wake of a ventilated hydrofoil is not very clearly understood. Also, these processes will depend upon the liquid flow, air ventilation rate and the downstream distance from the turbine. The detailed understanding of the bubble coalescence and breakup events in the wake as a function of these parameters will aid to the development of physical models for bubble size distribution.

There have been numerous reports on the bubble breakup phenomena [5 - 8], and similarly some studies have exclusively focused on coalescence of bubbles $[9,10]$. Many previous studies have reported the occurence of coalescence and breakup of bubbles or its effect on resulting bubble size distributions in different types of flow configurations such as air-sparged bubble columns [11-16], a two-dimensional packed bed [17], pipes [18-20], water jet [21] etc. However, to the best of our knowledge, there are hardly any studies conducted in the wake of a turbine hydrofoil blade. The flow physics in the bubbly wake of a hydroturbine is markedly different as compared to these other flow configurations often reported. Specifically, there are very few reports on the prediction of bubble size in a turbulent bubbly wake flow. The recent measurements in the turbulent bubbly wake and the oxygen transfer $[1,2]$ have employed a ventilated hydrofoil in a 
water tunnel for simulating the flow around a hydroturbine. However, a detailed insight into the physical processes in the wake and its effect on the resulting bubble size has not been provided. Therefore, in the current study, we conducted a detailed investigation into the breakup and coalescence processes in the wake of a ventilated hydrofoil and also proposed a theory to predict the maximum stable bubble size for dilute dispersions in a turbulent bubbly wake.

This paper is structured as follows: Section 2 provides the details for the experimental facility, the setup, the optical approach used to capture digital images and the image analysis technique employed in our study. Subsequently in Section 3, we present results and discussion on bubble size distributions in the wake and offer the insights into the coalescence and breakup mechanism involved, which is followed by a final conclusion in Section 4.

\section{Experimental Methodology}

\subsection{Experimental Setup}
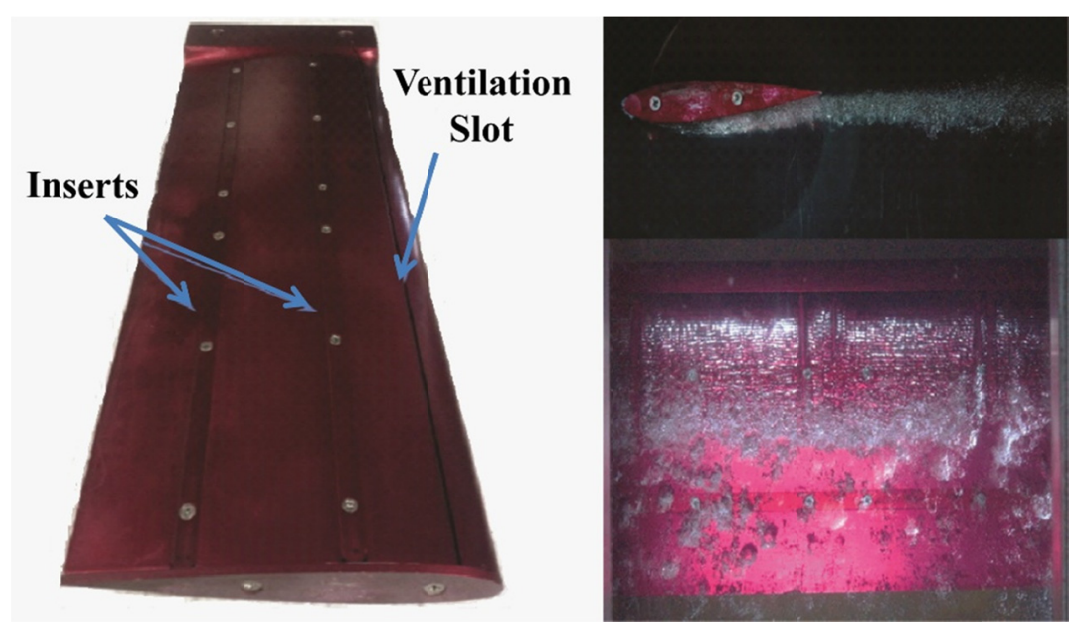

Figure 1: Details of the NACA0015 ventilated foil. Left: photo of suction side of hydrofoil. Right: Sample image of ventilated flow. Side view and bottom view of suction side. Adapted from [2].

The experiments are conducted in the SAFL high-speed water tunnel at the University of Minnesota. The tunnel has a horizontal test section of $1 \mathrm{~m}$ (Length) $\times 0.19 \mathrm{~m}$ (Width) $\times 0.19 \mathrm{~m}$ (Height) with three sides having Plexiglas walls for optical access. The tunnel is designed for 
cavitation and air ventilation studies, and is capable of operating at a maximum velocity of 20 $\mathrm{m} / \mathrm{s}$. A gas-collector dome in the tunnel provides for fast removal of large quantities of air bubbles generated during cavitation and ventilation experiments, enabling bubbly flow experiments for extended periods with little effect on test section conditions. A NACA 0015 hydrofoil similar to the study of [1] was employed in our experiments. During the experiments, the hydrofoil is installed at zero angle of attack in the test-section as shown in Figure 1. It has a span of $190 \mathrm{~mm}$ and a chord length (c) of $81 \mathrm{~mm}$ and a narrow spanwise slot that allows air to be injected into the flow near the leading edge of the hydrofoil, resulting in a narrow spanwise bubbly wake. The injection slot is $0.5 \mathrm{~mm}$ in thickness and the air exits the slot at an angle of $45^{\circ}$ to the hydrofoil chord. In order to make bubble size measurements, ventilation is restricted to a narrow $9.6 \mathrm{~mm}$ wide slot ( $5 \%$ of span) at the center of the span, thus ensuring that bubbles remain mostly within a narrow distance away from the centerline. As discussed in Karn et al. [1], the bubbles obtained in the current experiment setup provides a reasonable representation of the bubble population when the full span of the insert is ventilated. The air flow rates in the testsection is controlled by a mass flow controller, with uncertainty being less than $1 \%$ of the measured value. Uncertainty in the measurement of test-section velocity was less than $2 \%$ of the measured value.

\subsection{Experimental Techniques}

A Shadow Imaging Velocimetry (SIV) technique was used in our experiments, as has been reported previously by [1]. The SIV technique employs direct in-line volume illumination with a LED light source and an optical setup to produce a narrow depth-of-field for 2D plane imaging $[22,23]$. A pulsed LED light source from Innovative Scientific Solutions Inc. is used to illuminate the flow, which has flash rates up to $10 \mathrm{kHz}$ with a $5 \mu$ s pulse width and rise and fall 
times around $200 \mathrm{~ns}$. To ensure uniform back-lighting and to eliminate noise in the images, a light shaping diffuser is placed between the light source and the flow. A $1 \mathrm{~K} \times 1 \mathrm{~K}$ pixel Photron APX-RS camera with a $60 \mathrm{~mm}$ focal length lens is used for image acquisition.

In the SIV technique, two LED light pulses separated by small time duration are synchronized with camera exposure in order to obtain two consecutive (or, double) images. In our experiments, the time duration between two pulses varied between 100 and $230 \mu$ s depending upon the free stream velocity. Using the image pairs, the instantaneous velocity field of the bubbles is obtained through a commercial software (LaVision DaVis 7.2), for Particle Image Velocimetry (PIV). The field of view (FOV) of the captured images is approximately $60 \mathrm{~mm} \times 60 \mathrm{~mm}$ and such images are captured at five different downstream locations in the wake corresponding to $x / c=1.3,2.1$, 3.0, 3.7 and 4.7 as shown in Figure 2. The imaging system is calibrated prior to the start of the experiments using a $2.5 \mathrm{~mm} \times 2.5 \mathrm{~mm}$ calibration plate at the centerline of the test section. The use of the imaging lens in our experiments ensured that there are negligible distortions in our images, even at the edge of the FOV. Both pixel dimensions are $0.059 \mathrm{~mm}$ in all calibrated images. The image depth of field is determined to be approximately $15 \mathrm{~mm}$ at the sampling plane. The lateral spread in the bubble plume is measured at the measurement location and the calculated uncertainty in spanwise bubble location translates to a length scale (and calculated velocity) uncertainty of $1.6 \%$ near the foil and $3.2 \%$ at the most downstream location (i.e. 377 $\mathrm{mm}$ ). The bubble sizes are in the range of 2 to 68 pixels, which corresponds to a bubble diameter of 0.12 to $4 \mathrm{~mm}$. It is worth noting that the term 'size' and 'diameter' are used interchangeably in the paper. 
The water velocity in the experiments ranges from 3 to $10 \mathrm{~m} / \mathrm{s}$ with the air ventilation rate in the range of $1-3 \times 10^{-5} \mathrm{~kg} / \mathrm{s}$. The pressures inside the test-section and the air temperature under these conditions have been tabulated in Table 1.

\begin{tabular}{|c|c|c|c|}
\hline $\boldsymbol{V}(\mathrm{m} / \mathrm{s})$ & $\boldsymbol{Q}\left(\times 10^{-5} \mathrm{~kg} / \mathrm{s}\right)$ & $\boldsymbol{P ( k P a )}$ & $\boldsymbol{T}(\mathbf{K})$ \\
\hline 10 & 3.00 & 49.8 & 294 \\
\hline 10 & 1.00 & 49.5 & 294 \\
\hline 7.5 & 2.26 & 72.5 & 294 \\
\hline 7.5 & 1.00 & 72.7 & 294 \\
\hline 5 & 3.00 & 89.5 & 294 \\
\hline 5 & 2.50 & 89.8 & 294 \\
\hline 5 & 2.00 & 89.6 & 294 \\
\hline 5 & 1.50 & 89.6 & 294 \\
\hline 5 & 1.00 & 89.8 & 294 \\
\hline 3 & 1.00 & 98.0 & 294 \\
\hline
\end{tabular}

Table 1: Pressure in the test-section and the air temperature at each experimental condition.

Under each experimental condition, two sets of bubble images are recorded at 50 and 1500 frames/s, respectively. Each data set consists of 2000 images acquired over a duration of $40 \mathrm{~s}$. To examine the detailed dynamics of the bubbles in the wake, high speed imaging at $2000 \mathrm{frames} / \mathrm{s}$ is employed with an exposure time of $15 \mu$ s to prevent any blurring of bubbles in the images. 


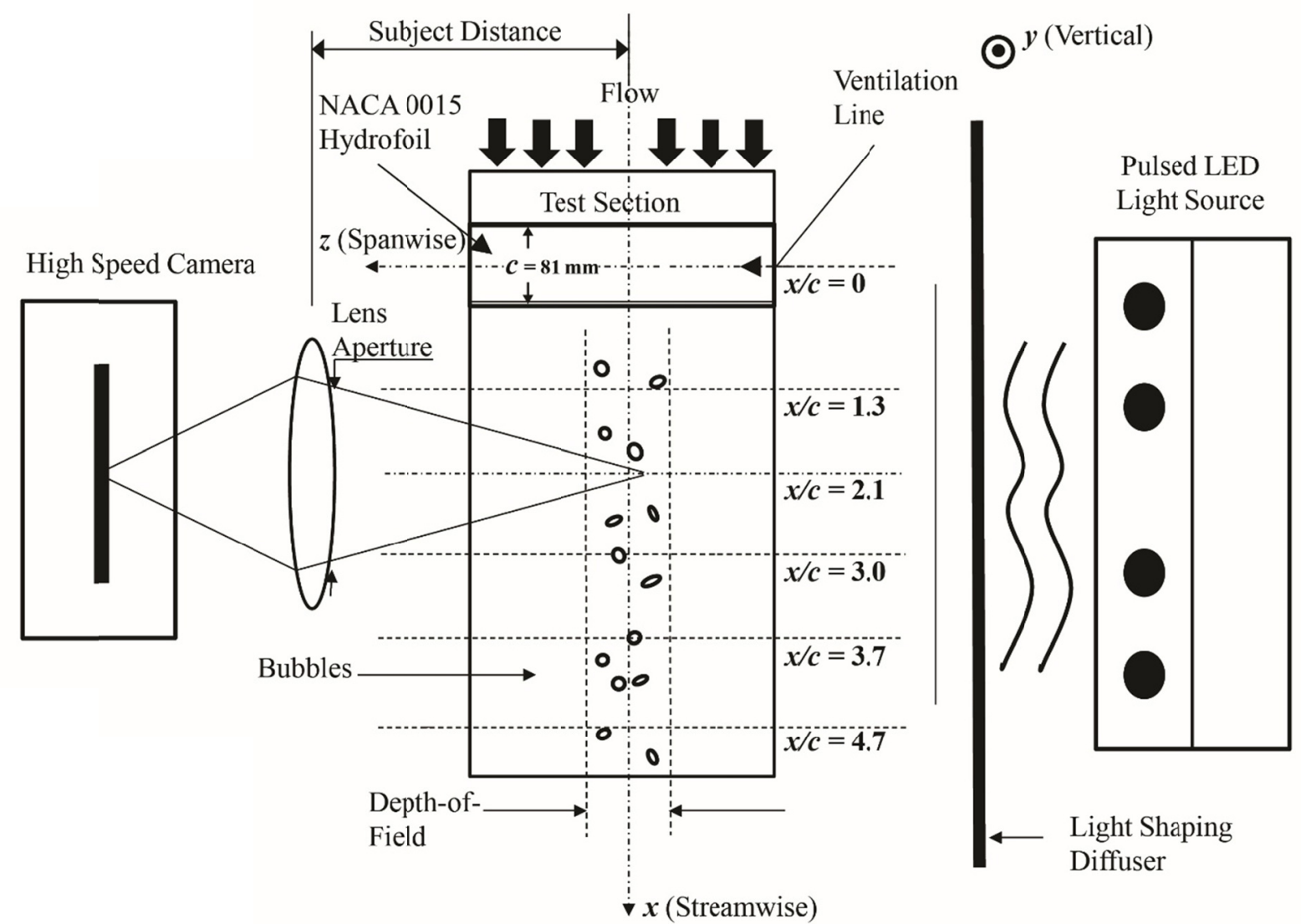

Figure 2: A schematic of the experimental setup showing five different locations of image capture in the wake of the ventilated hydrofoil. FOV of each imaging window is $60 \mathrm{~mm} \times 60 \mathrm{~mm}$.

\subsection{Image Analysis and Bubble Size Measurement}

The bubble images are analyzed using an algorithm which has been described in a previous paper by Karn et al. [24]. In this technique, the bubble regions are categorized based on size into tiny bubbles, intermediate-size bubbles and large bubbles/bubble clusters. The bubble clusters are further analyzed through a 'cluster-processing' step where these clusters are separated into individual bubbles. The projected areas of the bubbles are traced and assuming each bubble to be a generic ellipsoid, all the three radii of each bubble are estimated by assuming that the bubbles have an axisymmetric shape about the major axis. The uncertainty of the image analysis technique in measuring the bubble sizes is found to be size-dependent and is outlined in [24].The 
mean uncertainty in measuring the size of the bubbles varies from $6-10 \%$ at the smallest size to less than $2 \%$ for bubbles larger than $3.5 \mathrm{~mm}$ in size.

\section{Results and Discussion}

\subsection{Mean Bubble Statistics}

Experiments are performed to determine the mean bubble size statistics at ten different experimental conditions over four different water velocities $(U=10,7.5,5$ and $3 \mathrm{~m} / \mathrm{s})$ and varying air ventilation rates $\left(Q=0.50,1.00,1.50,2.00,2.50,3.00 \times 10^{-5} \mathrm{~kg} / \mathrm{s}\right)$. Table 2 below shows the mean bubble statistics for these different test cases at the farthest downstream location (i.e. $x / c=4.7$, referred to as 'reference location' hereafter). The average void fraction is obtained by processing the bubble images. As presented in Table 2, this average void fraction increases with increased air ventilation at a fixed liquid velocity. A number of experiments are carried out at a liquid velocity of $5 \mathrm{~m} / \mathrm{s}$, with air ventilation rates increasing by three times from $1.0 \times 10^{-5}$ $\mathrm{kg} / \mathrm{s}$ to $3.0 \times 10^{-5} \mathrm{~kg} / \mathrm{s}$ and the void fraction consequently increases from 0.032 to 0.065 . For a fixed air ventilation rate of $1.0 \times 10^{-5} \mathrm{~kg} / \mathrm{s}$, average void fraction increases from 0.028 to 0.047 as liquid velocity is increased from 3 to $10 \mathrm{~m} / \mathrm{s}$. This increment in void fraction can be explained based on the measurements of total number of bubbles in the flow and the resultant bubble size. In general, Table 1 shows that an increase in liquid velocity increases the number of bubbles and brings about a decrement in the size of the bubbles. This result suggests a production of smaller bubbles upon the increase in liquid velocity, resulting in increased bubble surface area, and thus void fraction. Further, Table 2 also presents the values of different characteristic diameters from our bubble size measurements. In our experiments, a more reliable estimate of the maximum stable bubble diameter is obtained from the cumulative size distributions. Accordingly, $d_{99}$ or 
$d_{99.8}$, or the bubble diameter that is larger than $99 \%$ and $99.8 \%$ respectively, of all the bubble diameters in the population, is chosen as a representative maximum bubble size.

\begin{tabular}{|c|c|c|c|c|c|c|c|c|}
\hline $\begin{array}{c}V \\
(\mathbf{m} / \mathbf{s})\end{array}$ & $\begin{array}{c}\boldsymbol{Q} \underset{\mathrm{kg} / \mathrm{s})}{(} \times 10^{-5} \\
\end{array}$ & $\begin{array}{c}\text { Average void } \\
\text { fraction, } \phi\end{array}$ & $\begin{array}{c}\text { Number of } \\
\text { bubbles, N/(1e6) }\end{array}$ & $\begin{array}{c}d_{99} \\
(\mathrm{~mm})\end{array}$ & $\begin{array}{c}d_{32} \\
(\mathrm{~mm})\end{array}$ & $C_{\mathrm{n}}$ & $\begin{array}{l}d_{99.8} \\
(\mathrm{~mm})\end{array}$ & $\begin{array}{l}d_{\max } \\
(\mathrm{mm})\end{array}$ \\
\hline 10 & 3.00 & 0.091 & 2.382 & \begin{tabular}{|l|}
0.88 \\
\end{tabular} & 0.54 & 0.61 & 0.95 & 0.95 \\
\hline 10 & 1.00 & 0.047 & 1.805 & 0.73 & 0.43 & 0.59 & \begin{tabular}{|l|}
0.78 \\
\end{tabular} & \begin{tabular}{|l|}
0.78 \\
\end{tabular} \\
\hline 7.5 & 2.26 & 0.059 & 1.325 & 0.96 & 0.60 & 0.62 & 1.03 & 1.03 \\
\hline 7.5 & 1.00 & 0.036 & 1.068 & 0.84 & 0.51 & 0.61 & \begin{tabular}{|l|}
0.90 \\
\end{tabular} & \begin{tabular}{|l|}
0.90 \\
\end{tabular} \\
\hline 5 & 3.00 & 0.065 & 0.782 & 1.27 & 0.81 & 0.64 & 1.38 & 1.38 \\
\hline 5 & 2.50 & 0.057 & 0.723 & 1.25 & 0.79 & 0.63 & 1.35 & 1.35 \\
\hline 5 & 2.00 & 0.050 & 0.677 & 1.22 & 0.77 & 0.63 & 1.31 & 1.31 \\
\hline 5 & 1.50 & 0.042 & 0.645 & 1.15 & 0.73 & 0.63 & 1.24 & 1.24 \\
\hline 5 & 1.00 & 0.032 & 0.573 & 1.08 & 0.68 & 0.63 & 1.16 & 1.16 \\
\hline 3 & 1.00 & 0.028 & 0.242 & 1.51 & 0.97 & 0.64 & 1.62 & 1.62 \\
\hline
\end{tabular}

Table 2: Bubble size characteristics obtained at different experimental conditions at the reference location. $N$ is the total number of bubbles at each downstream location in the entire dataset of 2000 images.

The Sauter mean diameter, $d_{32}$ is computed as:

$d_{32}=\sum_{i=1}^{N} d_{i}^{3} / \sum_{i=1}^{N} d_{i}^{2}$

Where $d_{i}$ is diameter of a single bubble and $N$ denotes the total number of bubbles in the population. Further, to characterize the bubble size distributions in our experiments, we compute the value of another coefficient $C_{\mathrm{n}}$ which is defined as the ratio of $d_{32}$ and $d_{99}$. $C_{\mathrm{n}}$ is an important design parameter and it can be used to estimate the corresponding value of $d_{32}$ (provided $d_{99}$ is known), which is important to determine interfacial area of dispersed phase, usually required for heat and mass transfer applications. As presented in Table 2, for the bubble populations in our experiments, $C_{\mathrm{n}}$ has an average value of 0.62 with minimal variations over all the experimental conditions. This value matches with that of 0.62 reported by Hesketh [16] for gas-liquid dispersions and 0.60 reported by Calabrese [25] for liquid-liquid dispersions. 


\subsection{Bubble Size Distribution at the Reference Location}

In general, bubble populations with a minimum bubble size are expected to show a lognormal distribution, as reported in the previous studies of Hesketh [16] and Razzaque [9]. Therefore, in order to further characterize the bubble populations in our study, we compare our bubble size distributions to standard log-normal distributions. It is found that generally the bubble size distributions at the reference location in our experiments indeed follow a log-normal pattern. The lognormal distribution function in number, $f(d)$ is given as

$f(d)=\frac{1}{\sqrt{2 \pi} \ln \sigma_{g}} \exp \left[-\frac{1}{2}\left(\frac{\ln \left(d / d_{g}\right)}{\ln \sigma_{g}}\right)^{2}\right]$

Where $d_{g}$ and $\sigma_{g}$ are the geometric mean size of the bubbles and geometric mean standard deviation, respectively and can be estimated from the raw data as follows:

$d_{g}=\left(\prod_{i=1}^{N} d_{i}\right)^{1 / N}$

$\log \sigma_{g}=\sqrt{\frac{\sum_{i=1}^{N}\left[\log d_{i}-\log d_{g}\right]^{2}}{N}}$

Table 3 below presents the value of $d_{g}$ and $\sigma_{g}$ obtained by fitting bubble size data to a lognormal distribution for different experimental conditions: 


\begin{tabular}{|c|c|c|c|c|}
\hline $\boldsymbol{V}(\mathrm{m} / \mathrm{s})$ & $\boldsymbol{Q}\left(\times 10^{-5} \mathrm{~kg} / \mathrm{s}\right)$ & $\boldsymbol{d}_{\mathbf{g}}$ & $\boldsymbol{\sigma}_{\mathbf{g}}$ & $\boldsymbol{C}_{\boldsymbol{n}}^{\prime}$ \\
\hline 10 & 3.00 & 0.30 & 1.66 & 2.26 \\
\hline 10 & 1.00 & 0.25 & 1.57 & 2.20 \\
\hline 7.5 & 2.26 & 0.32 & 1.70 & 2.28 \\
\hline 7.5 & 1.00 & 0.28 & 1.64 & 2.25 \\
\hline 5 & 3.00 & 0.43 & 1.83 & 2.29 \\
\hline 5 & 2.50 & 0.42 & 1.82 & 2.29 \\
\hline 5 & 2.00 & 0.41 & 1.81 & 2.29 \\
\hline 5 & 1.50 & 0.39 & 1.79 & 2.29 \\
\hline 5 & 1.00 & 0.36 & 1.76 & 2.29 \\
\hline 3 & 1.00 & 0.49 & 1.95 & 2.24 \\
\hline
\end{tabular}

Table 3: Fitting parameters used to fit bubble size distribution with a lognormal distribution at different experimental conditions. $C_{n}^{\prime}$ is defined in equation 5 .

From the above table, it can be observed that at a fixed liquid velocity, the change in air ventilation rate does not affect the mean geometric size or the width of the distribution. However, both $d_{g}$ and $\sigma_{g}$ decrease as the average water velocity is increased, i.e. the bubble size distribution becomes wider and shifts towards left with increasing average water velocity at a fixed air ventilation rate of $1.0 \times 10^{-5} \mathrm{~kg} / \mathrm{s}$. In the reported literature on bubble size distributions, the lognormal behavior of the bubble size distribution has been confirmed by the calculation of another parameter, $\boldsymbol{C}_{\boldsymbol{n}}^{\prime}$, which is defined as $\boldsymbol{C}_{\boldsymbol{n}}^{\prime}=d_{99.8} / d_{32}$. Further, based on lognormal distribution, it can be shown that $\boldsymbol{C}_{\boldsymbol{n}}^{\prime}$ is only a function of the width of distribution $\sigma_{g}$ :

$\boldsymbol{C}_{\boldsymbol{n}}^{\prime}=\frac{d_{99.8}}{d_{32}}=\exp \left[-\left(2.5 \ln ^{2} \sigma_{g}-2.88 \ln \sigma_{g}\right)\right]$

For the obtained values of $\sigma_{g}$ from bubble size distribution in our experiments, the value of $\boldsymbol{C}_{\boldsymbol{n}}^{\prime}$ is calculated and shown in Table 2. These values are close to 2.2 as reported before for lognormal bubble size distributions [9].

The calculation of $\boldsymbol{C}_{\boldsymbol{n}}^{\prime}$ parameter has important practical implications. Provided $d_{99.8}$ is known, it can yield an estimate of the value of Sauter mean diameter of a bubble population, which is very important for numerous practical applications concerning heat and mass transfer. As a 
consequence, the estimation of Sauter mean diameter essentially reduces to the determination of $d_{99.8}$. Based on Table 2, it can be pointed out that the values of $d_{99.8}$ and the maximum stable bubble size in the flow, $d_{\max }$ are almost equal, as evidenced in our experiments. Assuming that $d_{99.8}$ represents the maximum stable bubble size in the flow, the determination of $d_{32}$ and $d_{\max }$ can be correlated. The estimation of $d_{\max }$ in a flow has been the subject of research for several studies beginning with that of Hinze [5]. Many authors such as Levich [26], Sevik and Park [6] and Hesketh [16] have investigated the stable bubble size due to bubble breakup in low void fraction flows. In our study, we employ Hinze's theory to predict the maximum bubble size in the turbulent hydrofoil wake flow, which is presented in following section.

\subsection{Stable bubble size in the turbulent hydrofoil wake}

To estimate the maximum stable bubble size due to the bubble breakup, we choose a location in the wake closest to the hydrofoil. Compared with other measurement locations, the most breakup events associated with turbulent fluctuations are expected at this location (please refer to the following section for detailed discussions on the dominant regimes of bubble breakup and coalescence). The maximum stable bubble size in a flow-field is determined by a balance between turbulent pressure fluctuations and surface tension forces $[5,6,16,26,27]$. Turbulent fluctuations tend to deform or break the bubble while surface tension forces resist such deformation. Considering a balance between these two forces, Hinze defined a critical Weber number as follows:

$$
W e_{\text {Crit }}=\frac{\tau}{\sigma / d_{\max }}
$$

Where $\tau$ is the stress on the bubble surface due to turbulent fluctuations in the continuous phase, $\sigma$ is the interfacial tension, $\rho_{d}$ and $\rho_{c}$ are the dispersed and continuous phase densities, 
respectively and $d_{\max }$ is the diameter of the largest stable bubble in the turbulent flow field. Hinze characterized the dynamic pressure force of the continuous phase as

$$
\tau=\rho_{c} \overline{v^{2}}
$$

Where the mean-square spatial fluctuating velocity term, $\overline{v^{2}}$ has been estimated by Batchelor [28] for homogeneous isotropic turbulence as

$\overline{v^{2}}=2\left(\epsilon d_{\max }\right)^{2 / 3}$

Where $\epsilon$ denotes the energy dissipation rate per unit mass. In [16], Hesketh approximated the local energy dissipation rate using the average energy dissipation $\bar{\epsilon}$ calculated from mean turbulent velocity profile in the flow.

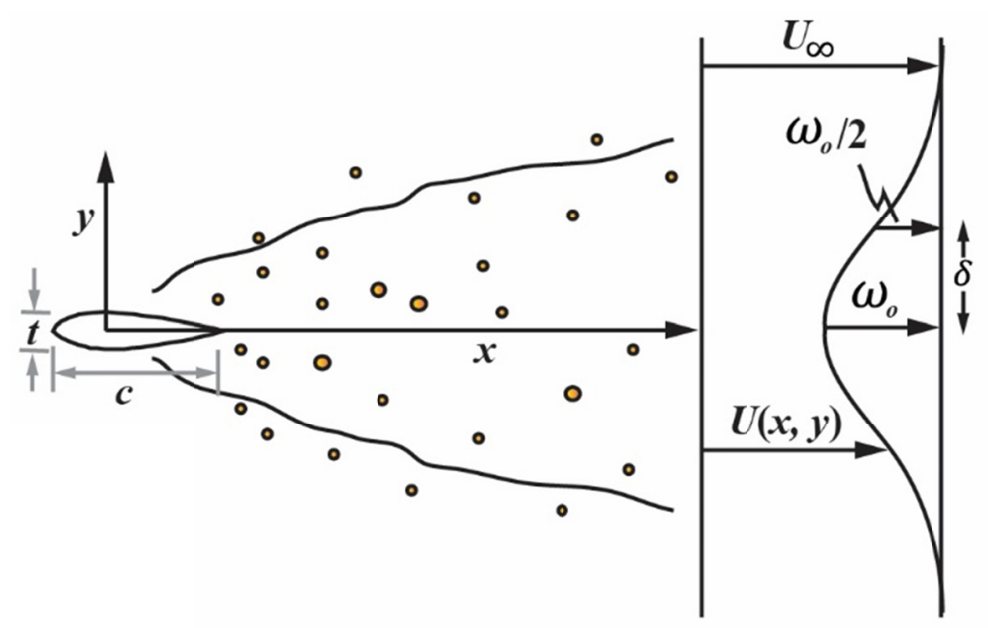

Figure 3: A schematic of the two dimensional bubbly wake.

As shown in Figure 3, the liquid velocity at any location in the wake is a function of both $x$ and $y$ coordinates. The streamwise liquid velocity outside the wake is designated as $U_{\infty}$ and the velocity at the centerline of the wake is denoted by $U_{o}(x)$. The difference $U_{\infty}-U(x, y)$ is the velocity deficit, $\omega$, and the maximum velocity deficit at a particular location is $\omega_{o}\left[=U_{\infty}-\right.$ $U(x, 0)]$. The distance between the wake centerline and the location where the velocity deficit is 
half the maximum is denoted by $\delta$. At a sufficient downstream distance away from the hydrofoil in the wake, the mean and turbulence quantities attain self-preservation. Hence, $\omega / \omega_{o}$ is solely a function of $y / \delta$ and the dependence upon the streamwise direction comes into play because of the streamwise variation of $\omega_{o}(x)$ and $\delta(x)$. According to Sreenivasan [29], the ensemble average of the energy dissipation rate can be expressed as:

$$
\langle\varepsilon\rangle=0.035 \frac{\omega_{o}^{3}}{\delta}
$$

Combining equations (6) to (9); an expression for maximum stable bubble size can be obtained as:

$$
d_{\text {max }}=\epsilon^{-\frac{2}{5}}\left(\frac{\sigma W e_{c}}{2 \rho_{w}}\right)^{\frac{3}{5}}
$$

For the dispersions in air-water fluid pair, a critical Weber number of 1.1 is used, as reported by a number of prior studies $[9,16,22]$. As a result, $d_{\max }$ can be expressed as:

$$
d_{\max }=2.67\left(\frac{\delta}{\omega_{o}^{3}}\right)^{2 / 5}\left(\frac{\sigma}{\rho_{w}}\right)^{3 / 5}
$$

Further, $\delta$ and $\omega_{o}$ at a particular downstream distance in the wake can be calculated from the expressions given below, which are deduced based on the velocities obtained from previous PIV measurements conducted at SAFL in the wake of a NACA0015 hydrofoil at different downstream locations:

$$
\begin{aligned}
& \frac{\omega_{o}(x)}{U_{\infty}}=0.12\left(\frac{x}{c}\right)^{-\frac{1}{2}} \\
& \frac{\delta(x)}{c}=0.07\left(\frac{x}{c}\right)^{\frac{1}{2}}
\end{aligned}
$$

In our study, the above expressions were found to match with the velocity field derived from the bubble velocities. Note that although bubbles in a flow do not accurately trace the flow, yet the 
mean velocities of the bubbles are an accurate representation of the actual mean velocity field, provided that the bubbles are not accelerating.

Figure 4 below presents the comparison of the measured maximum bubble size with the largest stable bubble size, $d_{\max }$ predicted by theory. Although there is significant deviation in the experimental data, yet the mean values of the maximum bubble size closely matches the prediction from Hinze's theory. In general, the proposed theory has a slightly steeper variation in bubble size with respect to freestream velocity compared to the experimental data. It must also be pointed out that the flow conditions chosen for this comparison are the ones that correspond to a low ventilation flowrate of $1.0 \times 10^{-5} \mathrm{~kg} / \mathrm{s}$ and at a location closest to the hydrofoil. This ensures that measured bubble sizes are a result of bubble breakup processes and are not influenced by the occurrence of coalescence in the bubbly wake.

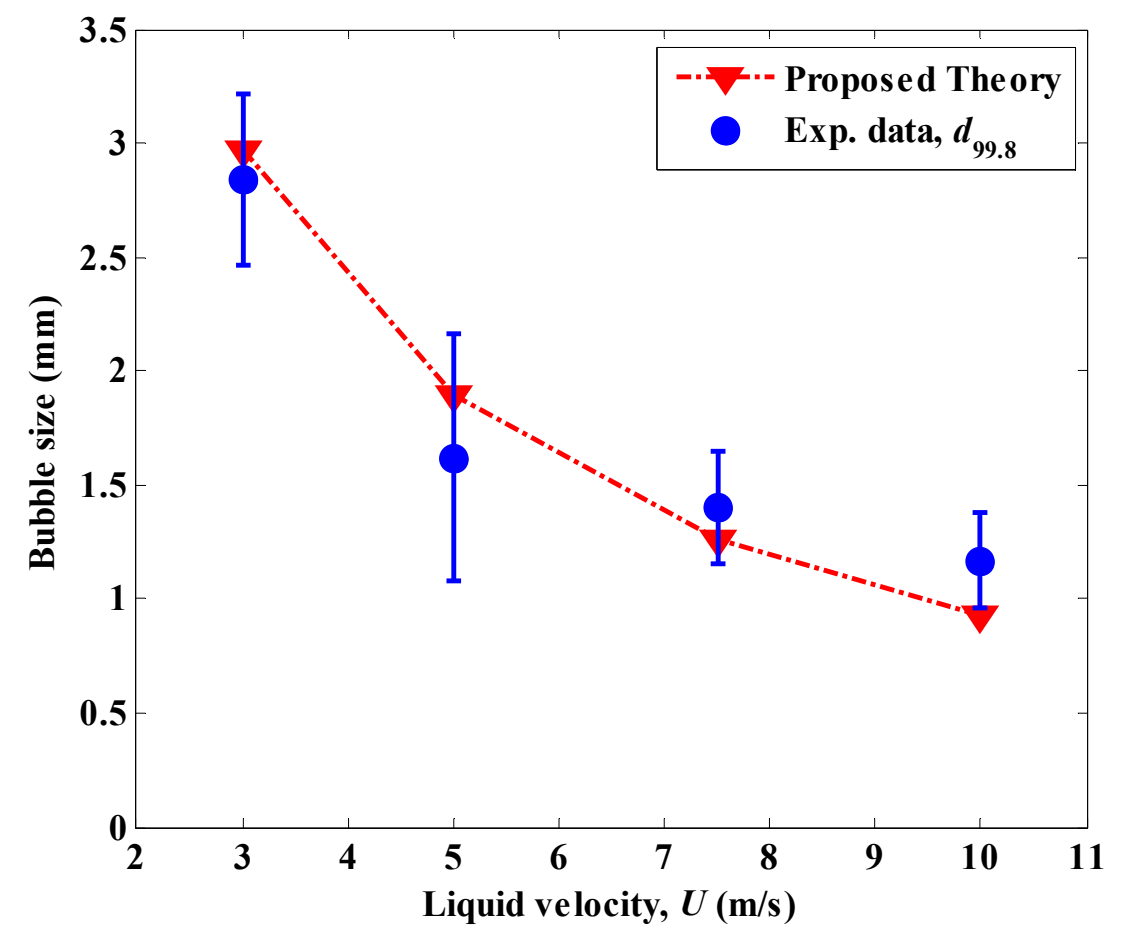

Figure 4: Comparison of maximum bubble size as proposed by our bubble dispersion theory and as observed at the viewing location closest to the hydrofoil. All experiments correspond to an air flow rate of $1.0 \times 10^{-5} \mathrm{~kg} / \mathrm{s}$. The error-bars denote one standard deviation in the chosen experimental dataset at each flow condition. 


\subsection{Variation of mean bubble statistics in the wake}

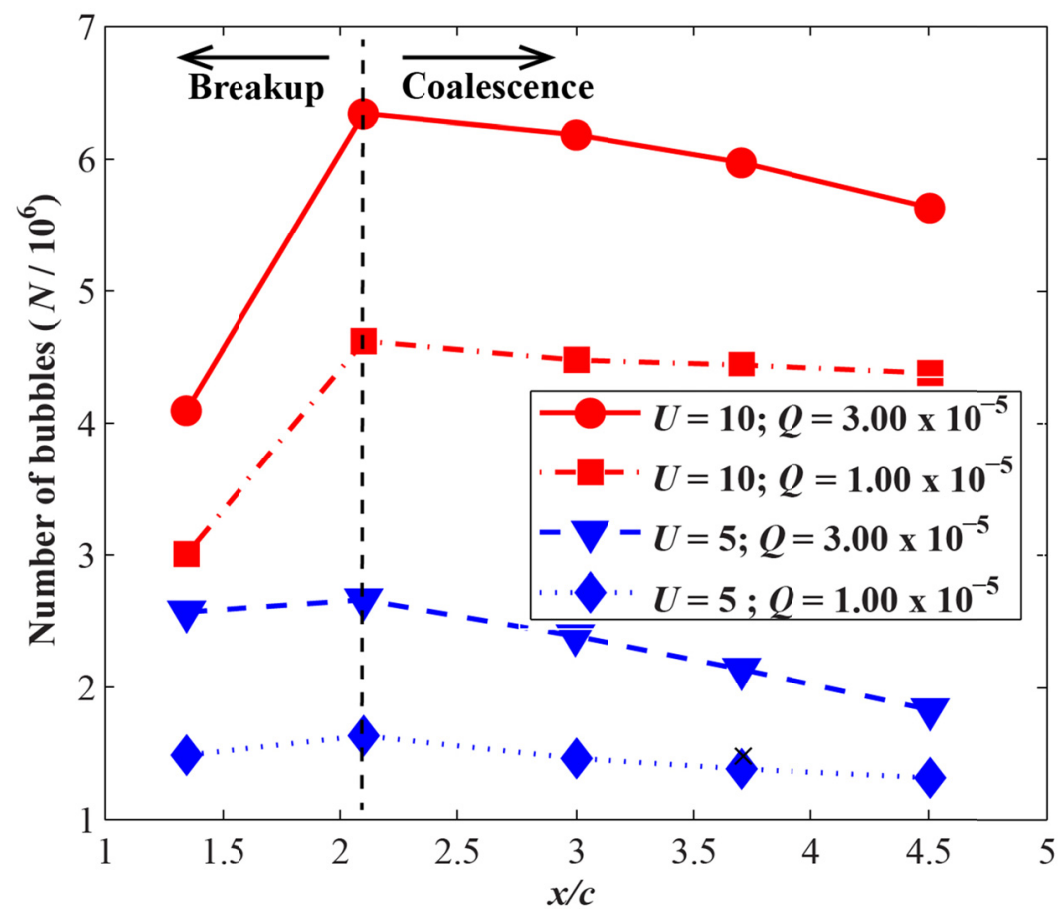

Figure 5: Number of bubbles passing through different downstream locations in the wake. $N$ is the total number of bubbles at a particular downstream location in the entire dataset of 2000 images. The units of $U$ and $Q$ are $\mathrm{m} / \mathrm{s}$ and $\mathrm{kg} / \mathrm{s}$, respectively.

The mean bubble statistics in the hydrofoil wake are investigated at different downstream locations in the bubbly wake by examining the number of bubbles passing through each viewing section for the entire dataset for each experimental condition. Figure 5 above shows the number of bubbles present at each viewing section at four different experimental conditions comprising two different water velocities and air ventilation rates. As the figure shows, for all the experimental cases, the number of bubbles increases from $x / c=1.3$ to $x / c=2.1$, and starts decreasing upon moving downstream from $x / c=2.1$. The change in the number of bubbles at different downstream locations is related to the relative significance of competing breakup and coalescence events in the wake. It is observed that the predominance of the breakup and coalescence events in the wake varied at different locations. There exists a break-up dominant regime close to the hydrofoil, whereas coalescence dominates farther in the wake. In addition, 
the slopes of the curves in the breakup-dominant and coalescence-dominant regimes are governed by liquid velocity and air ventilation rates, respectively. The occurrence of distinct breakup and coalescence regimes in the hydrofoil wake is further confirmed by the measurements of $d_{32}$ at different locations as shown in Table 4:

\begin{tabular}{|c|c|c|c|c|c|}
\hline $\boldsymbol{V}(\mathrm{m} / \mathrm{s})$ & $\boldsymbol{Q}\left(\times 10^{-5} \mathrm{~kg} / \mathrm{s}\right)$ & $d_{32 \mid x / c}=1.3(\mathrm{~mm})$ & $d_{32 \mid x / c}=2.1(\mathrm{~mm})$ & $d_{32 \mid x / c}=3.0(\mathrm{~mm})$ & $d_{32 \mid x / c}=4.7(\mathrm{~mm})$ \\
\hline 5 & 1.00 & 0.85 & 0.63 & 0.67 & 0.68 \\
\hline 5 & 3.00 & 0.97 & 0.71 & 0.76 & 0.81 \\
\hline 10 & 1.00 & 0.82 & 0.42 & 0.42 & 0.43 \\
\hline 10 & 3.00 & 1.02 & 0.51 & 0.50 & 0.54 \\
\hline
\end{tabular}

Table 4: Variation of $d_{32}$ at different downstream locations in the hydrofoil wake for selected test-cases

As Table 4 shows, for all the test cases, $d_{32}$ decreases from $x / c=1.3$ to $x / c=2.1$, and increases upon moving downstream from $x / c=2.1$ to $x / c=4.7$, confirming the distinct occurrence of bubble breakup close to the hydrofoil and coalescence at a farther downstream location in the wake. It is noteworthy that no appreciable change in bubble size is observed between $x / c=3.7$ and $x / c=4.7$. 


\subsubsection{The Coalescence-dominant regime.}

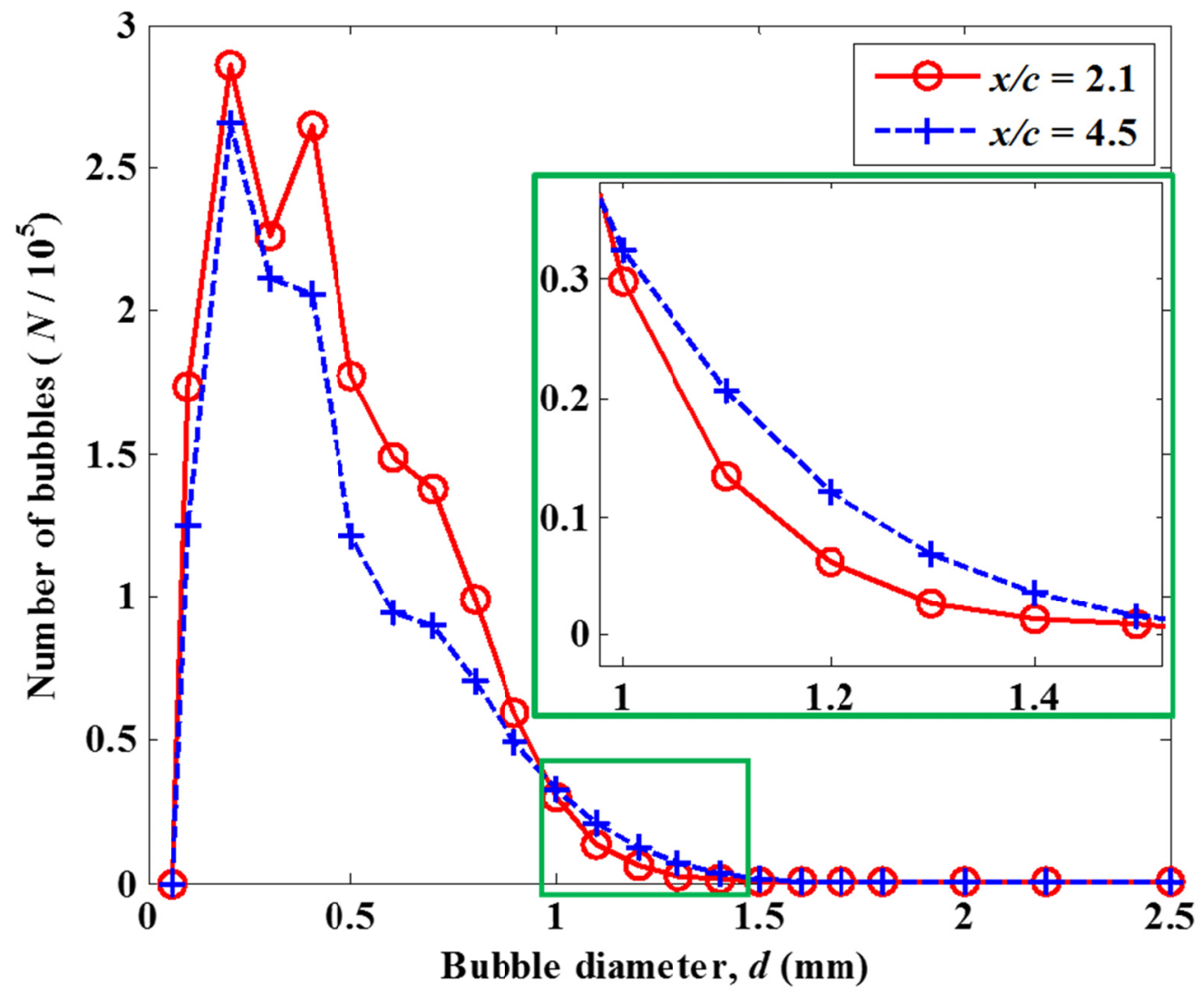

Figure 6: Number of bubbles distribution at two downstream locations in the wake showing the occurrence of bubble coalescence. Both the distributions are for $V=5 \mathrm{~m} / \mathrm{s}$ and $Q=3.0 \times 10^{-5} \mathrm{~kg} / \mathrm{s}$. Inset is a magnified view of the green rectangle shown on the plot.

Figure 6 presents BSDs at $x / c=2.1$ and $x / c=4.7$, respectively. As shown in the figure, upon moving from $x / c=2.1$ to $x / c=4.7$, the number of bubbles less than $1 \mathrm{~mm}$, especially between $0.3-0.9 \mathrm{~mm}$, decrease while there is an increase for bubbles of size larger than $1 \mathrm{~mm}$ (see inset). This result suggests that between these two downstream locations in the wake, the bubbles in 0.3 - $0.9 \mathrm{~mm}$ size range undergo coalescence, producing bubbles in the larger size range. The resulting larger bubbles do not further undergo coalescence because of the reduced efficiency of coalescence at larger sizes. Previous reports in the literature have shown that the coalescence efficiency between two bubbles depends strongly on the size of the bubbles involved [10]. As pointed out by Liao and Lucas [10], coalescence process consists of three stages: (1) Collision of 
bubbles leading to entrapment of a liquid film between them; (2) Bubbles staying in contact with each other till the liquid film drains to a critical thickness; (3) Rupture of the liquid film resulting in coalescence. The duration of the collision is limited by the velocity fluctuations existing in the flow, and coalescence occurs only when the time of interaction of two bubbles $\left(t_{\text {contact }}\right)$ is sufficient to allow for the intervening film to drain out to a critical rupture thickness, i.e., $t_{\text {contact }}$ exceeds the film drainage time $\left(t_{\text {drainage }}\right)$. According to Coulaloglou [30], the coalescence efficiency is given by exp $\left(-t_{\text {drainage }} / t_{\text {contact }}\right)$, and drainage time has been reported to vary as the square of the equivalent diameter of the two colliding bubbles [31-33]. Thus, the smaller the coalescing bubbles, less is the drainage time and greater the coalescence efficiency. This observation has been confirmed by our experiments in the horizontal turbulent hydrofoil wake. Figure 7 shows two separate cases of bubble interaction illustrating the dependence of bubble coalescence upon bubble size. As the figure shows, the smaller bubbles coalesce rapidly as they come into contact (referred to as 'immediate coalescence' in [8]), whereas the larger bubbles need to remain in contact for a much longer period for coalescence to take place. As shown in Figure $7 \mathrm{~b}$, even after the formation of a liquid film between the two bubbles, the velocity fluctuations around the bubble might separate them as the bubbles acquire sufficient energy. The dependence of coalescence efficiency on size explains the preferential coalescence of bubbles in the size range of $0.3-0.9 \mathrm{~mm}$ compared with that of larger bubbles. Further, as reported by Crabtree and Bridgwater [14], in our experiments coalescence of bubbles was observed to occur along the diagonals (especially when a trailing bubble is about to overtake a leading bubble) and such events were much more abundantly present than the occurrence of bubble coalescence upon head-on interaction of the bubbles. 


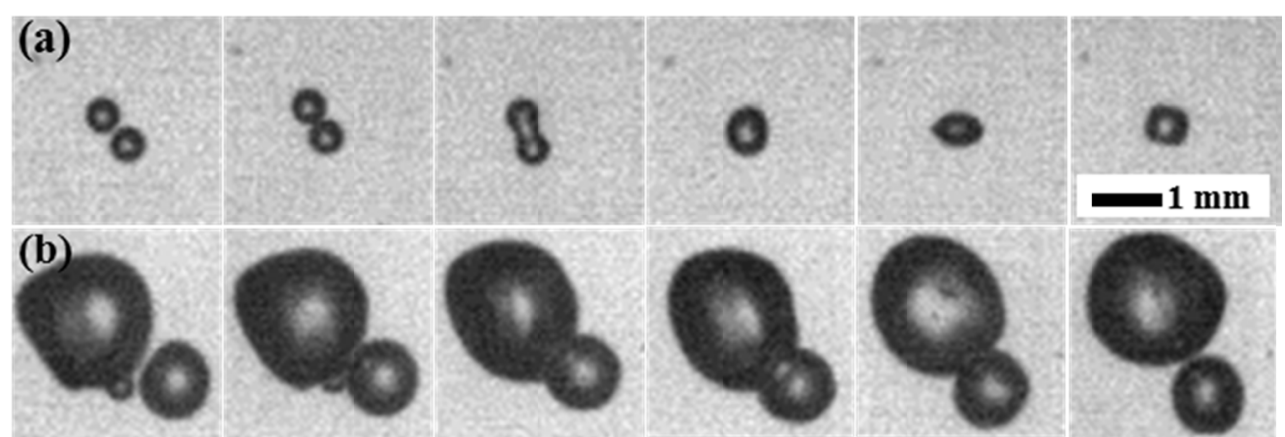

Figure 7: (a) Immediate coalescence of two small bubbles. (b) Contact of bubbles, film rupture and separation of bubbles. The scale bar applies to all the images.

The careful investigation of BSD in the coalescence-dominant regime also reveals the presence of extremely large individual bubbles with diameters above $4 \mathrm{~mm}$. The generation of such large size individual bubbles through a successive coalescence route seemed unlikely. Hence, to explain the occurrence of these extremely large bubbles, we carried out systematic and careful investigations into the formation of such large size bubbles through high speed imaging of the bubbly wake. Our observations revealed the occurrence of a 'cluster-coalescence' mechanism, whereby more than two bubbles can approach and coalesce simultaneously. Figure 8 shows an example of such process. As the figure shows, in a cluster-coalescence process, many bubbles simultaneously approach together and concomitant multiple film formation and film drainage events occur, generating a large size bubble.
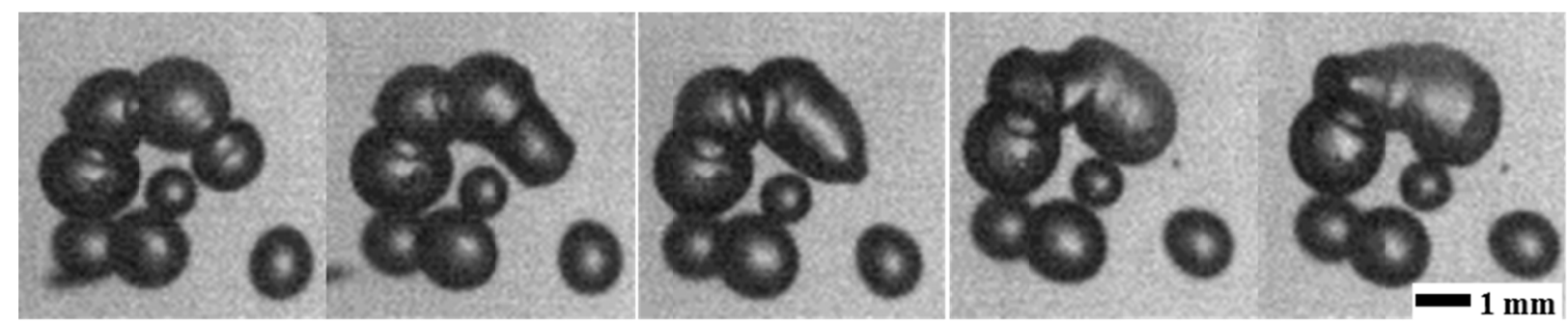

Figure 8: Occurrence of 'cluster coalescence'. The scale bar applies to all the images.

Finally, we focus our attention on the small size range of the BSD as presented in Figure 6. It can be observed that at both the downstream locations, there is a large number of small size bubbles (smaller than $0.5 \mathrm{~mm}$ ) present in the flow. This is somewhat surprising since the small bubbles 
might end up coalescing by virtue of their extremely high coalescence efficiency. In the reported literature on gas-liquid systems in coalescence-dominant regime, there are only a few studies which report the presence of significant number of very small size bubbles in addition to large bubbles in the flow $[34,35]$. Martin [34] proposed that such small bubbles may be generated by re-shedding from the rim of very large bubbles and may survive coalescence as a result of their small size among much larger bubbles. In our experiments, it is observed that the flow conditions favoring coalescence and a consequent increase in $d_{32}$ also showed a simultaneous rise in the number of small size bubbles upon moving downstream in the wake. Although this appears counter-intuitive, yet it seems to suggest a strong link between the generation of large bubbles through coalescence and the number of these small bubbles. To get an insight into this, we carefully investigated the high speed videos of hundreds of binary coalescence events. In some of these events, it is observed that post-coalescence, the coalesced bubble undergoes severe shape oscillations forming a spherical or spherical-cap bubble, and sometimes undergoing a minor pinch-off. Figure 9 shows such a process of coalescence-mediated breakup of small bubbles $[15,36]$. As the figure shows, rapid deformation during coalescence produces lobes at the ends of the original bubbles, and these lobes can be successively broken into microbubbles bubbles due to Rayleigh instability. Note that although the formation of these lobes are invariably observed in a coalescence event, often the pinched off microbubble is obscured by the coalescing bubble or large bubble present nearby. 


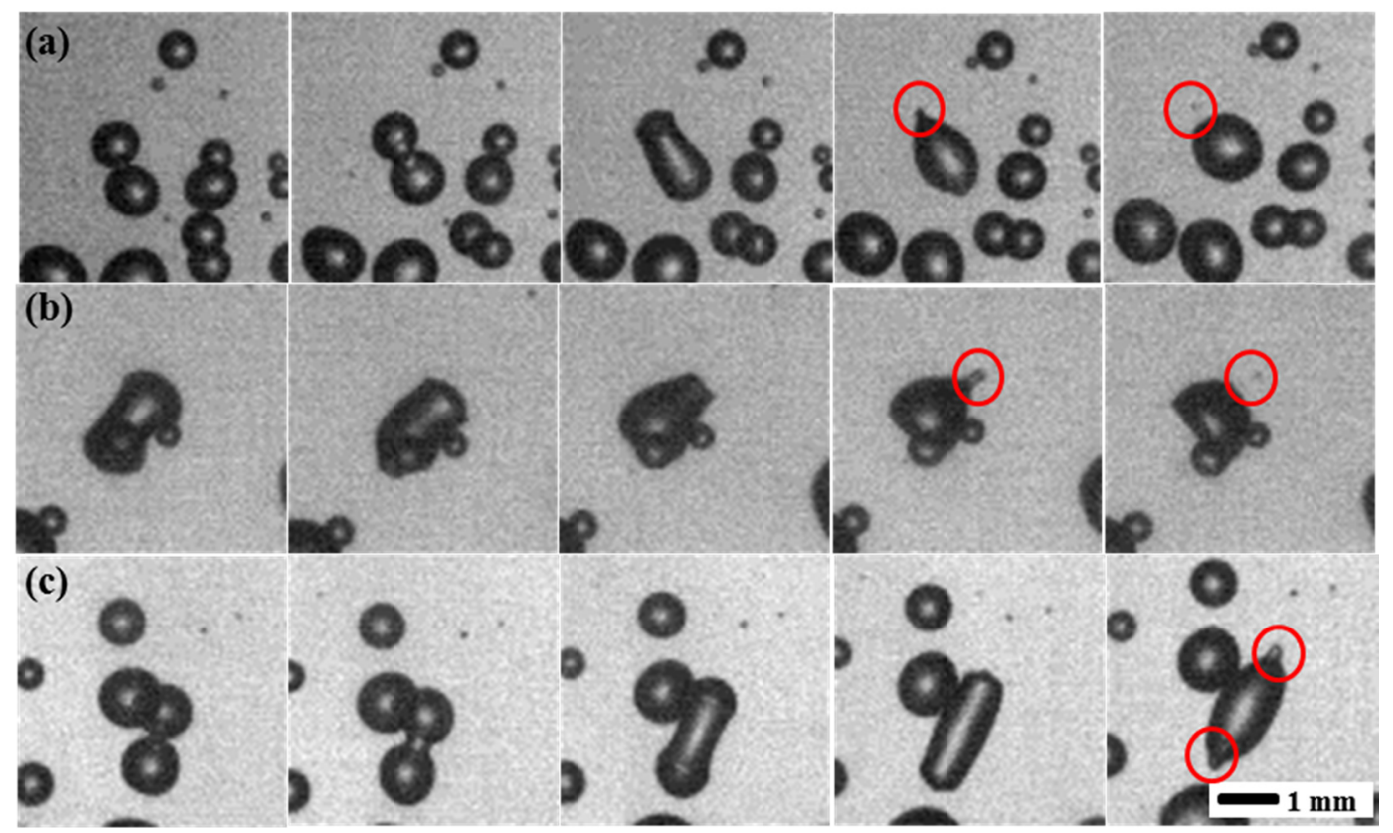

Figure 9 (a), (b), (c): Three different cases of occurrence of 'coalescence-mediated breakup'. The scale applies to all the images.

In summary, the contact and collision of bubbles is the very basis of coalescence process [10] and such collisions are augmented by the presence of increasing number of bubbles in the flow. In other words, an increase in air ventilation rate augments the occurrence of coalescence events in the coalescence-dominant-regime of the wake, leading to an increase in Sauter mean diameter of the bubbles. Since a larger air ventilation increases the void fraction in the flow, the Sauter mean diameter shows a significant dependence on the void fraction. In our experiments, the bubble Sauter mean diameter can be characterized as function of void fraction $\varphi$ at a fixed average liquid velocity as follows:

$d_{32} \propto \sqrt{\varphi}+c$

where $c$ is a constant for air-water fluid pair and the system properties. Similar dependence of bubble size upon the void fraction has been reported previously for coalescence-dominant regime by other authors $[9,37]$. Figure 10 shows this dependence of $d_{32}$ upon the void fraction. As the figure shows, $d_{32}$ increased from $0.67 \mathrm{~mm}$ at $\varphi=3 \%$ to $0.81 \mathrm{~mm}$ at $\varphi=6 \%$. Further, there is an 
excellent agreement between the measurements of Sauter mean diameter at different void fractions from our experiments and the fitting expression as defined in equation (14).

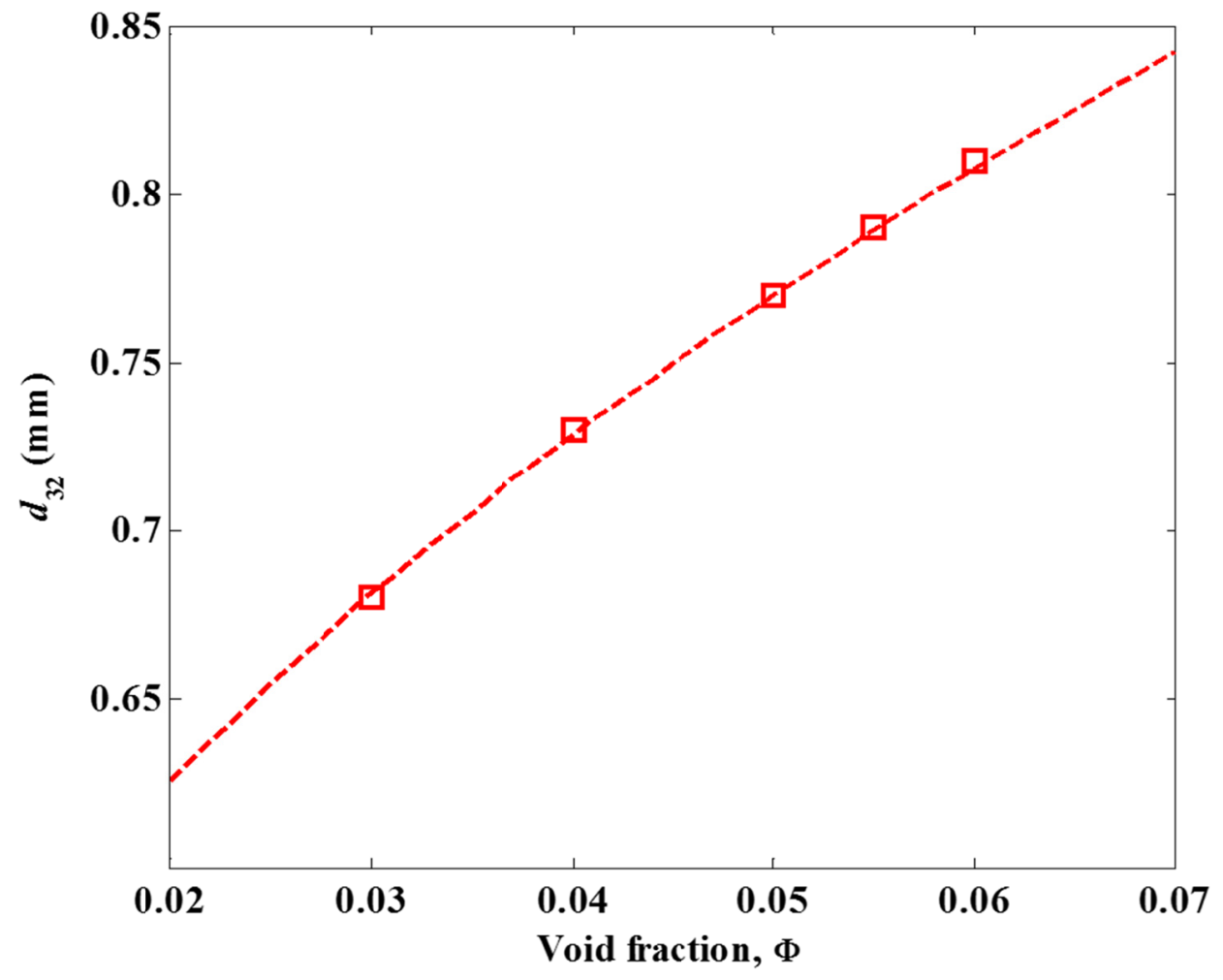

Figure 10: Dependence of $d_{32}$ upon void fraction, $\varphi$, at a fixed continuous phase velocity of $5 \mathrm{~m} / \mathrm{s}$. The squares show the values from the experiments while the dotted line shows the fit according to equation (14). 


\subsubsection{The breakup-dominant regime.}

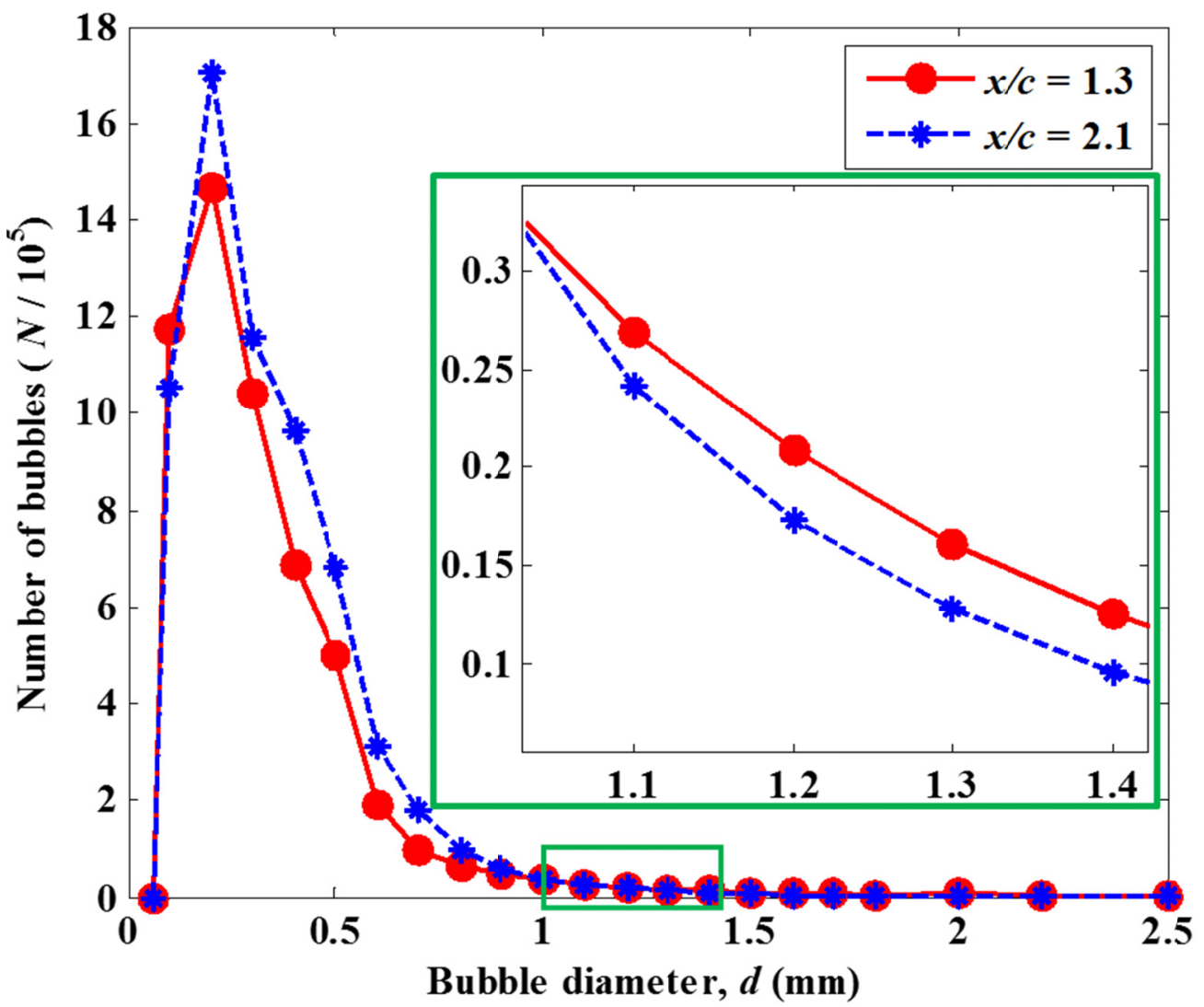

Figure 11: Number of bubbles distribution at two downstream locations in the wake showing the occurrence of bubble breakup. Both the distributions are for $V=10 \mathrm{~m} / \mathrm{s}$ and $Q=3.0 \times 10^{-5} \mathrm{~kg} / \mathrm{s}$. Inset is a magnified view of the green rectangle shown on the plot. The axis labels for the inset are the same as the original plot.

To further investigate the breakup phenomena, the number of bubbles distribution at $x / c=1.3$ and $x / c=2.1$ is plotted as shown in Figure 11. As the figure presents, the location closer to the hydrofoil is marked by a greater number of large size bubbles (larger than $1 \mathrm{~mm}$, as shown in the inset). On the other hand, at a further downstream location of $x / c=2.1$, a larger number of smaller bubbles are present in the flow. This suggests that the larger bubbles present at $x / c=1.3$ split to produce these smaller bubbles. Our observations indicate that large size bubbles readily undergo deformation and thus are more likely to split into small size bubbles. High speed videos of the bubbles in the breakup-dominant-regime shows that the bubble surface is in continuous irregular motion caused by the turbulent eddies interacting with its surface. These deformations 
are mainly observed in the large-diameter $(>1 \mathrm{~mm})$ ellipsoidal bubbles, which have a lower internal pressure and thus, are easily deformed. Further, these deformations occur when the diameter of a bubble is larger than the Kolomogorov length scale, and is of the order of the size of energy-containing eddies. The eddies that are larger than the bubble size merely carry the bubbles, whereas the eddies much smaller than the bubbles do not have ample energy to break it [12]. But, the deformation caused by the eddies of a similar size as the bubble sets up motion in the gas phase and destabilizes the bubble due to an increase in surface energy. When the amplitude of the oscillation becomes large, it makes the bubble surface unstable. Then bubble surface starts to deform and stretch in one direction, giving rise to a neck that contracts further to cause breakage. Figure 12 below shows three examples of such breakup events as observed in the breakup-dominant regime.

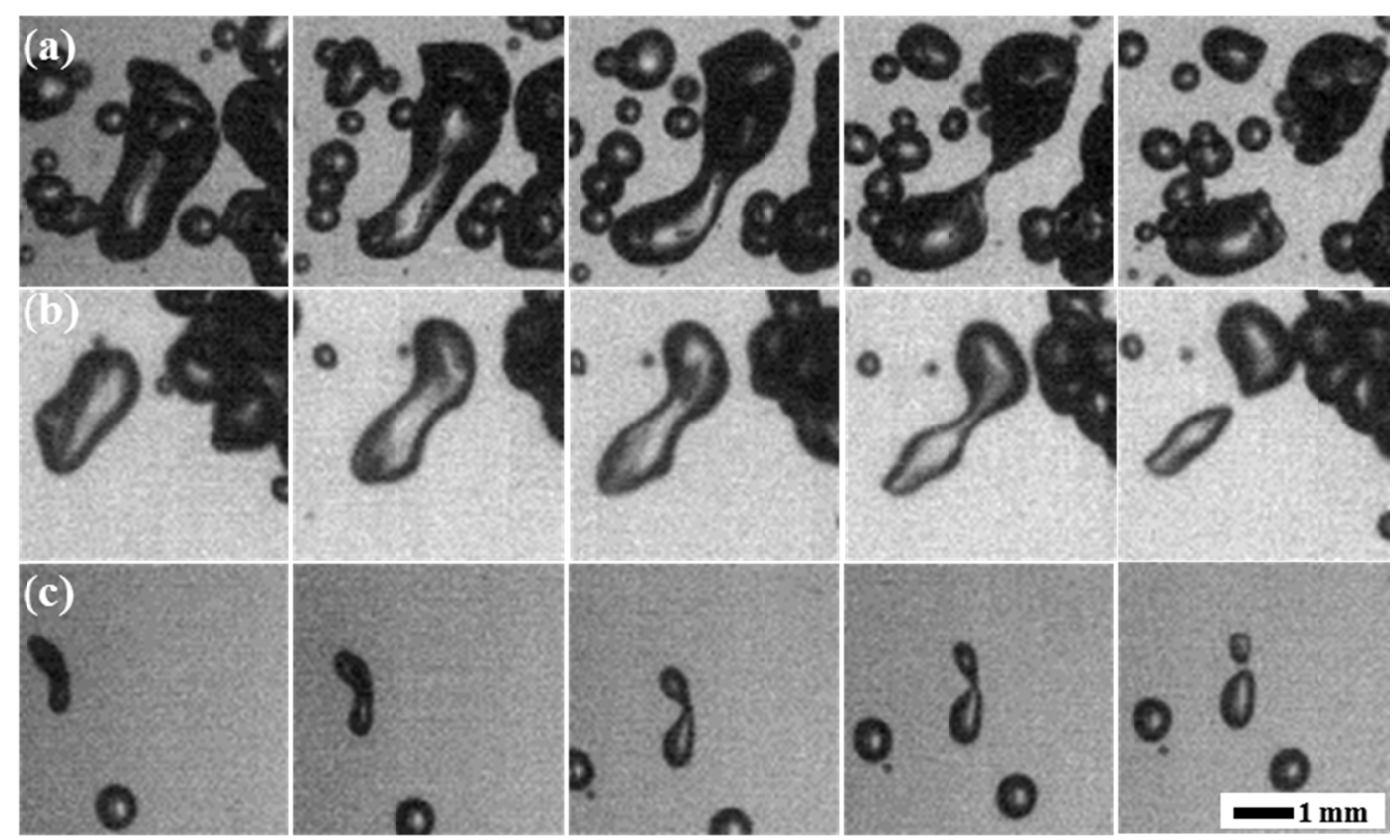

Figure 12: (a), (b) and (c): Three different cases of bubble breakup caused by turbulent pressure fluctuation or by bubble-eddy collision. The scale applies to all the three cases.

The above images show an initial bubble ranging from $1-3 \mathrm{~mm}$ undergoing breakup into two individual bubbles of almost equal size. There is no generation of small daughter bubbles 
observed in these images. This is not surprising since Hesketh et al. [7] also experimentally found that bubble breakup is essentially always binary in horizontal pipe flow. However, our high-speed imaging observations revealed another mechanism of generation of these microbubbles through bubble collision and subsequent tearing as shown in Figure 13. As the figure shows, a small bubble of $0.2 \mathrm{~mm}$ in diameter enters into the wake region of a leading bubble that is more than $2 \mathrm{~mm}$ in length. In the wake of the larger bubble, the small bubble undergoes acceleration, and finally collides with the preceding bubble at a speed higher than the liquid velocity. Such acceleration of a small bubble caused in the wake of a larger bubble has been discussed before by Bilicki and Kestin [38]. In some cases, such a collision may cause tearing of the leading bubble and the teared portion of the bubble can further get sheared to microbubbles observed in the images.
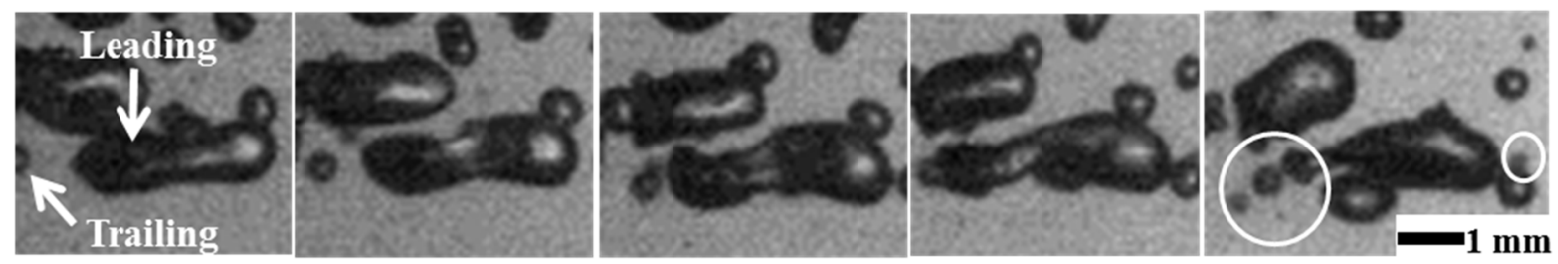

Figure 13: Bubble breakup caused by bubble tearing. Flow is from left to right. The image sequences are from left to right, and top to bottom row.

As the air gets entrained into the wake, it breaks down into small bubbles because of continuous phase hydrodynamics and interfacial interactions. Such breakup can be caused by turbulent velocity fluctuations, bubble collisions, viscous shear stresses, interfacial instability or other shear effects [8]. However, in our experiments, apart from the coalescence-mediated breakup, the breakup of bubbles can be primarily attributed to turbulent velocity fluctuations, bubble-eddy collisions in the flow and bubble tearing resulting from bubble collisions. Our observations do not show a significant occurrence of coalescence events in the breakup dominant regime. Due to high turbulent pressure fluctuations in the near-wake of the hydrofoil, there is significant bubble 
breakup. In this breakup-dominant regime, where coalescence effects are not dominant, our proposed theory of bubble dispersion (refer to section 3) can predict maximum bubble sizes quite accurately.

\section{Conclusions}

In this paper, some insights into the breakup and coalescence phenomena occurring in the horizontal turbulent bubbly wake of a ventilated hydrofoil are presented. The bubble images in the bubbly wake are captured at five different downstream locations through a shadow imaging technique and the bubble images are processed through an image-analysis technique proposed by Karn et al [24]. The experiments are carried out at ten different flow conditions with liquid velocity varying in the range of $3-10 \mathrm{~m} / \mathrm{s}$ and air ventilation rates spanning $0.5-1.5 \times 10^{-5} \mathrm{~kg} / \mathrm{s}$. For the bubble size data obtained at these flow conditions, a dispersion theory to predict the bubble size is presented for dilute dispersions in a turbulent wake flow. The proposed dispersion theory is in a reasonably good agreement with the bubble size measured from the experimental data in the breakup dominant regime at low ventilation rates. For the bubble population in our experiments, the parameter $C_{\mathrm{n}}\left(=d_{32} / d_{99}\right)$ obtained experimentally has a mean value of 0.62 , which matches well with the value predicted by prior studies, both for gas-liquid dispersions [16] and liquid-liquid dispersions [26]. Further, bubble sizes from our experiments fit a lognormal distribution. This is confirmed by the good match of the value of parameter $\boldsymbol{C}_{\boldsymbol{n}}^{\prime}$, which can be expressed solely in terms of the width of the bubble size distribution. It is found that the obtained ratio of 2.2 matches perfectly with the previous reports of lognormal bubble size distributions [9].

Next, the number of bubbles and Sauter mean diameter is analyzed at different downstream locations to explore the occurrence of bubble breakup or coalescence. Based on the number of 
bubbles, it is observed that breakup dominates in the wake till a downstream distance of $x / c=2.1$ (breakup-dominant regime) while coalescence is predominant farther down in the wake (coalescence-dominant regime). It is understood that near the hydrofoil, the velocity fluctuations of the underlying turbulence of the jet results in deformation forces that exceed the confinement forces due to surface tension leading to more pronounced bubble breakup. The breakup continues while the bubbles are convected downstream to regions of lower dissipation rates, where breakup effects are superseded by the occurrence of coalescence.

Through high-speed imaging, we observed that coalescence process is augmented by increase in number of collisions between bubbles, which increases with number of bubbles in the flow, or the air ventilation rate. With an increase in air ventilation, the bubbles undergo successive coalescence causing an increment in Sauter mean diameter. Based on our experimental data, an expression for $d_{32}$ is proposed in terms of the void fraction in the flow at a fixed continuous phase velocity. The BSD at two different locations in the coalescence-dominant regime show that bubbles lesser than $1 \mathrm{~mm}$ undergo coalescence to produce larger bubbles. Further, it is also observed that small bubbles undergo "immediate coalescence" whereas the larger bubbles need to remain in contact for a longer time to coalesce. The presence of the extremely large bubbles (above $4 \mathrm{~mm}$ in diameter) is attributed to a phenomenon called "cluster coalescence". The presence of a large concentration of microbubbles in coalescence-dominant regime is found to be linked with the coalescence process through a 'coalescence-mediated breakup' mechanism. As far as breakup-dominant regime is concerned, two possible mechanisms are considered most important contributors to the bubble breakup. The breakup caused by the turbulent velocity fluctuations begins with a deformation on a large size bubble, stretches in one direction, giving rise to a neck that contracts to cause bubble breakage. In our experiments, such breakup events 
are always observed to be binary and no generation of microbubbles is observed, consistent with the assertion of Hesketh [7] for a horizontal pipe flow. Instead, the generation of these microbubbles can be attributed to a bubble collision and tearing process. In the wake of a large leading bubble, a small bubble might accelerate and collide with the leading bubble tearing away a portion of it, resulting in microbubbles observed in BSD.

Overall, this study provides the bubble size distributions obtained in the turbulent bubbly wake of a ventilated hydrofoil at different downstream locations in the wake. The BSDs are supported by actual observations of breakup and coalescence obtained from high speed imaging. And finally, expressions are provided for the prediction of Sauter mean diameter at different flow conditions, both in the breakup-dominant and coalescence-dominant regimes. It is hoped that these insights into breakup and coalescence will aid the development of a model of bubble size and help researchers improve CFD simulations of AVTs.

\section{Acknowledgements}

The authors gratefully acknowledge support by Department of Energy Contract \# DEEE0005416, ALSTOM Renewable Power Canada Inc. and the Office of Naval Research United States (Program manager, Dr. Ronald Joslin) under Grant \# N000140910141.

\section{References}

[1] A. Karn, C. Ellis, J. Hong, R.E.A. Arndt, Investigations into the turbulent bubbly wake of a ventilated hydrofoil: moving towards improved turbine aeration techniques. Exp. Therm. Fluid Sci. 64 (2015a) 186-195.

[2] A. Karn, G. M. Monson, C. Ellis, J. Hong, R.E.A. Arndt, J. S. Gulliver, Mass transfer studies across ventilated hydrofoils: A step towards hydroturbine aeration. Int. J. Heat Mass Trans. 87 (2015b) 512-520.

[3] C.E. Bohac, R.J. Ruane, Solving the dissolved oxygen problem, Hydro Rev. 9(1) (1990) 6273. 
[4] P.A. March, T.A. Brice, M.H. Mobley, J.M. Cybularz, Turbines for solving the DO dilemma, Hydro Rev., 11(1) (1992) 30-36.

[5] J.O. Hinze, Fundamentals of the hydrodynamic mechanism of splitting in dispersion processes, AIChE J. 1 (3) (1955) 289-295.

[6] M. Sevik, S.H. Park, The splitting of drops and bubbles by turbulent fluid flow, J. Fluid Eng. 95 (1973) 53-60.

[7] R.P. Hesketh, A.W. Etchells, T.W.F. Russell, Bubble breakage in pipeline flow, Chem. Eng. Sci. 46 (1991) 1-9.

[8] Y. Liao, D. Lucas, A literature review of the theoretical models for drop and bubble breakup in turbulent dispersions, Chem. Eng. Sci. 64 (2009) 3389-3406.

[9] M.M. Razzaque, A. Afacan, S. Liu, K. Nandakumar, et al, Bubble size in coalescence dominant regime of turbulent air-water flow through horizontal pipes, Intl. J. Multiphase Flow 29 (2003) 1451-1471.

[10] Y. Liao, D. Lucas, A literature review on mechanisms and models for the coalescence process of fluid particles, Chem. Eng. Sci. 65(2010) 2851-2864.

[11] E. Olmos, C. Gentric, Ch. Vial, G. Wild, N. Midoux, Numerical Simulation of multiphase flow in bubble column reactors. Influence of bubble coalescence and breakup, Chem. Eng. Sci. 56 (2001) 6359-6365.

[12]A. Laari, I. Turunen, Experimental determination of the bubble coalescence and break-up rates in a bubble column reactor, The Canadian J. of Chem. Eng. 81 (2003) 395-401.

[13] B.W. Atkinson, G.J. Gameson, A.V. Nguyen, G.M. Evans, P.M. Machniewski, Bubble breakup and coalescence in a plunging liquid jet bubble column, Can. J. Chem. Eng. 81 (2003) 519-527.

[14] J.R. Crabtree, J. Bridgwater, Bubble coalescence in viscous liquids, Chem. Eng. Sci. 26 (1971) 839-851.

[15] K.L. Tse, T. Martin, C.M. McFarlane, A. W. Nienow, Small bubble formation via a coalescence dependent break-up mechanism, Chem. Eng. Sci. 58 (2003) 275-286.

[16] M. J. Prince, H.W. Blanch, Bubble coalescence and break-up in air-sparged bubble columns, AIChE J.36 (10) (1990) 1485-1499.

[17] J. Daeseong, S. T. Revankar, Effect of coalescence and breakup on bubble size distributions in a two-dimensional packed bed, Chem. Eng. Sci 65.14 (2010) 4231-4238. 
[18] R.P. Hesketh, T.W.F. Russell, A.W. Etchells, Bubble size in horizontal pipelines, AIChE J. 33 (4) (1987) 663-667.

[19] P. Andreussi, A. Paglianti, F. S. Silva, Dispersed bubble flow in horizontal pipes, Chem. Eng. Sci. 54 (1999) 1101-1107.

[20] Th. Frank, et al., Validation of CFD models for mono-and polydisperse air-water two-phase flows in pipes, Nuclear Engineering and Design 238(3) (2008) 647-659.

[21] C. Martínez-Bazán, J. L. Montanes, J. C. Lasheras, On the breakup of an air bubble injected into a fully developed turbulent flow. Part 2. Size PDF of the resulting daughter bubbles, J. Fluid Mech. 401 (1999) 183-207.

[22] D. Bröder, M. Sommerfeld, An advanced LIF-PLV system for analysing the hydrodynamics in a laboratory bubble column at higher void fractions, Exp. Fluids 33(6) (2002) 826-837.

[23] L.P. Goss, J. Estevadeoral, J.W. Crafton, Velocity measurements near walls, cavities, and model surfaces using Particle Shadow Velocimetry (PSV), In: Proceedings of the 22nd International Congress on Instrumentation in Aerospace Simulation Facilities, 2007.

[24] A. Karn, C. Ellis, R. Arndt, J. Hong, An integrative image measurement technique for dense bubbly flows with a wide size distribution, Chem. Eng. Sci. 122 (2015c) 240-249.

[25] R.V. Calabrese, T.P.K. Chang, P. T. Dang, Drop Breakup in Turbulent Stirred-Tank Contactors. I, AIChE J. 32 (1986) 657-666.

[26] V.G. Levich, Physicochemical hydrodynamics, Vol. 689, Prentice-Hall Englewood Cliffs, NJ, 1962.

[27] A.N. Kolmogorov, On the breakage of drops in a turbulent flow, Dokl. Akad. Nauk. SSSR 66 (1949) 825-828.

[28] G. Batchelor, Theory of Homogeneous Turbulence, Cambridge, 122, 1959.

[29] K.R. Sreenivasan, The energy dissipation in turbulent shear flows, Symposium on developments in Fluid dynamics and Aerospace Engineering, Interline publishers, Bangalore, India, 1995, 159-190.

[30] C.A. Coulaloglou, Dispersed phase interactions in an agitated flow vessel, Ph.D. Dissertation, Illinois Institute of Technology, Chicago, 1975.

[31] R.H. Davis, et al., The lubrication force between two viscous drops, Physics of Fluids A 1 (1989) 77-81. 
[32] A.K. Chesters, The modeling of coalescence processes in fluid-liquid dispersions: A review of current understanding, Chemical Engineering Research and Design: transactions of the Institution of Chemical Engineers: Part A 69 (1991) 259-270.

[33] H. Luo, Coalescence, breakup and liquid circulation in bubble column reactors, Ph.D. Dissertation, The Norwegian Institute of Technology, Trondheim, 1993.

[34] T. Martin, Gas dispersion with radial and hydrofoil impellers in fluids with different coalescence characteristics, Ph.D. Thesis, University of Birmingham, UK, 1996.

[35] V. Machon, A. W. Pacek, A. W. Nienow, Bubble sizes in electrolyte and alcohol solutions in a turbulent stirred vessel, Chemical Engineering Research and Design 75(3) (1997) 339-348.

[36] M. Ohnishi, H. Azuma, J. Straub, Study on secondary bubble creation induced by bubble coalescence, Adv. Space Res. 24 (10) (1999) 1331-1336.

[37] P.H. Calderbank, Physical rate processing in industrial fermentation. Part I: the interfacial area in gas-liquid contacting with mechanical agitation, Trans. IChemE 36 (1958) 443-463.

[38] Z. Bilicki, J. Kestin, Transition criteria for two-phase flow patterns in vertical upward flow, Intl. J. Multiphase Flow 13 (1987) 283-294. 